Revista Destaques Acadêmicos, Lajeado, v. 8, n. 3, 2016. ISSN 2176-3070 DOI: http://dx.doi.org/10.22410/issn.2176-3070.v8i3a2016.1056 www.univates.br/revistas

\title{
O ACOLHIMENTO AO USUÁRIO COM RISCO CARDIOVASCULAR EM UMA UNIDADE DE SAÚDE DA FAMÍLIA
}

\author{
Marina Líbio ${ }^{1}$, Cássia Regina Gotler Medeiros²
}

\begin{abstract}
Resumo: Este artigo teve como objetivo analisar a prática do acolhimento ao usuário com risco de desenvolver Doença Cardiovascular (DCV) em uma unidade de Estratégia de Saúde da Família (ESF). As DCV têm se apresentado como a principal causa de morte no Brasil. É comprovado que estratégias que promovam a saúde reduzem a morbimortalidade pelas doenças crônicas não transmissíveis (DCNT), desde que se realizem ações de controle dos fatores de risco. Trata-se de um estudo de caráter observatório não participante, com abordagem qualitativa, que utilizou um diário de campo preenchido no momento em que o evento era observado, orientado por um instrumento com os fatores de risco para DCV definidos pelo Ministério da Saúde (MS). Os resultados demonstraram que o risco de desenvolver DCV é identificado parcialmente no acolhimento. A abordagem no acolhimento ao usuário com risco de desenvolver DCV ficou restrita a programar uma consulta médica e os cuidados estabelecidos foram de orientação para controle dos fatores de risco.
\end{abstract}

Palavras-chave: Acolhimento. Doença crônica. Estratégia saúde da família.

\section{INTRODUÇÃO}

As DCNT aparecem em primeiro lugar como causa de morte, o que significou 63\% dos óbitos no mundo em 2008 e 72\% das causas de mortes para o Brasil. As DCNT alcançam fortemente classes pobres da população e grupos vulneráveis (BRASIL, 2011). É comprovado que estratégias que promovam a saúde reduzem a morbimortalidade pelas DCNT, desde que se realizem ações no controle dos fatores de risco das DCNT (MALTA, 2012).

Nesse contexto, as DCV apresentam-se como principal causa de morte no Brasil, embora a mortalidade provocada por essas doenças tenha reduzido

1 Graduanda do curso de Enfermagem da Univates.

2 Possui graduação em Enfermagem e Obstetrícia pela Universidade de Passo Fundo (1986) e doutorado em Enfermagem pela Universidade Federal do Rio Grande do Sul (2013). 
no decorrer dos anos, ainda produzem custos enormes em relação a internações hospitalares. Dentre os fatores de risco para as DCV, está o tabagismo, consumo abusivo de álcool, inatividade física e consumo de alimentos com alto teor de gordura e densidade energética (IBGE, 2014).

Conforme Mendes (2012) a atenção exclusiva à agudização das condições crônicas, faz com que muitas vezes fatores que são determinantes sociais, riscos biopsicológicos e que estão interligados aos hábitos e aos estilos de vida do indivíduo, e que deveriam servir como base para uma atenção primária em saúde (APS) e proporcionar uma maior qualidade de vida, sejam negligenciados.

As situações e problemas de saúde, cada vez mais, apresentam-se com uma maior variabilidade, podendo ser desde uma situação de menor ou maior complexidade, fazendo com que os serviços de saúde tenham maiores desafios para contemplar toda demanda. Dessa forma, acolher os usuários torna-se de vital importância e abrange uma atenção mais significativa e com um olhar mais envolvente e responsável, o que auxilia nas necessidades de cada um (BRASIL, 2013b). O acolhimento é um dos conceitos que norteiam a Política Nacional de Humanização (PNH) e está como uma das diretrizes para atuar dentro da mesma. Acolher é distinguir o que a outra pessoa carrega como legítima e ímpar necessidade de saúde. $\mathrm{O}$ acolhimento deve aparecer e manter a relação entre equipes/serviços e usuários/ populações. Como relevante das práticas de saúde, o acolhimento se faz com escuta qualificada pelos trabalhadores com os usuários e propicia para os usuários o acesso à tecnologias apropriadas dependendo da sua necessidade (BRASIL, 2013a).

É necessário não limitar o conceito de acolhimento ao problema da demanda. $\mathrm{O}$ acolhimento na porta de entrada só ganha sentido se somarmos com o processo de produção de saúde, como algo que valoriza a relação e que, portanto, é capaz de ser apreendido e trabalhado em todo e qualquer encontro no serviço de saúde (BRASIL, 2009). Acolher de maneira adequada uma consulta de demanda espontânea implica sanar a necessidade atual e programar o acompanhamento de sua condição crônica. Dessa maneira, toda a ocorrência de uma situação aguda deve ser avaliada, levando em consideração alguns aspectos, tais como: uso das medicações prescritas, utilizadas, doses e horários; o consumo alimentar; a atividade física; o uso de álcool, tabaco e outras drogas; e fatores de conflitos e outros estresses emocionais (BRASIL, 2014).

Incluso no conceito atual de prevenção cardiovascular, mais relevante do que condicionar o indivíduo para o grupo de portadores de hipertensão arterial sistêmica (HAS), é distingui-lo em termos de seu risco cardiovascular. Perceber que a prevenção quando designada no conceito de risco cardiovascular tem maior efetividade para traçar planos preventivos, em função de somar os riscos decorrentes de diversos fatores (MATTOS, 2011). 
Os dados do Plano de Reorganização da Atenção à Hipertensão Arterial de 2001 constatou uma prevalência deste fator na população brasileira acima de 40 anos de idade de $36 \%$. O que significa que aproximadamente, mais de 15 milhões de brasileiros são portadores de HAS. Isso chama a atenção para buscar e trabalhar estratégias de atenção integral, de forma mais precoce no decorrer do ciclo da vida, intervindo e fazendo a prevenção do aparecimento da HAS e evitando agravamentos. É um desafio trabalhar com ações no cuidado integral, buscando o conhecimento científico e os avanços tecnológicos para trabalhar com a população (BRASIL, 2006a). Nesse aspecto, conhecer como é a prática do acolhimento ao usuário com risco de desenvolver DCV em uma ESF, é uma preocupação e responsabilidade enquanto pesquisadora e como futura trabalhadora da área. Partilhar as experiências e o que for analisado pode gerar aprendizagem para qualificar o cuidado.

Identificar medidas que permitam um atendimento que promova a saúde, a prevenção, ou que minimize os riscos é um desafio na elaboração deste estudo. Pretende-se observar o acolhimento na ESF para ampliar a qualidade dos atendimentos de saúde, por isso a questão que se colocou para essa observação é: "como é a prática do acolhimento ao usuário com risco de desenvolver DCV em uma unidade de saúde da família?".

Este artigo buscou verificar se o risco de desenvolver DCV é identificado no acolhimento, como é realizada a abordagem no acolhimento ao usuário com risco de desenvolver DCV, qual é o cuidado estabelecido com o usuário em risco de desenvolver DCV e comparar os processos de cuidado ao usuário com risco de desenvolver DCV na ESF, com o que preconiza o MS.

\section{METODOLOGIA}

Este estudo tem caráter observatório não participante, com abordagem qualitativa. Para Laville (1999, p. 176), a pesquisa de observação permite que o investigador tenha "um modo privilegiado de contato com o real, pois é observando que nos situamos, orientamos nossos deslocamentos, reconhecemos as pessoas, emitimos juízos sobre elas". De acordo com Goldim (2000, p. 133): "A pesquisa qualitativa é essencialmente descritiva e tem como perspectiva principal a visão de processo".

O campo de ação foram duas ESF de um município localizado no Vale do Taquari, Rio Grande do Sul. É um município predominantemente urbano, com área rural restrita. Possui aproximadamente 78.486 habitantes segundo dados do IBGE (2015). A ESF I abrange dois bairros, com 3.612 usuários e a ESF II abrange três bairros, com 3.566 usuários adstritos.

A coleta dos dados ocorreu no período de 07/03/2016 a 31/03/2016, quando foi observada a prática do acolhimento às pessoas com risco de desenvolver DCV, conforme a demanda do serviço da ESF. Foi usado como instrumento de pesquisa um diário de campo, preenchido no momento em 
que o evento era observado, orientado por um instrumento com os fatores de risco para DCV definidos pelo MS (BRASIL, 2006a): História familiar de doença arterial coronariana (DAC) prematura (familiar $1^{\circ}$. grau sexo masculino $<55$ anos e sexo feminino <65 anos); Homem $>45$ anos e mulher $>55$ anos; Tabagismo; Hipercolesterolemia (LDL-c elevado); Hipertensão arterial sistêmica; Diabetes melittus; Obesidade (IMC > $30 \mathrm{~kg} / \mathrm{m}^{2}$ ); Gordura abdominal; Sedentarismo; Dieta pobre em frutas e vegetais; Estresse psicossocial. Estes eram identificados a partir do relato do usuário no momento do acolhimento, o que determinava sua inclusão no estudo. Dessa forma, foram selecionados e observados 59 acolhimentos de pessoas com risco de desenvolver DCV.

Todos os participantes assinaram o Termo de Consentimento Livre e Esclarecido em duas vias após detalhada explicação sobre a pesquisa. O projeto foi aprovado pelo Comitê de Ética em Pesquisa da UNIVATES, em 10/02/2016, sob o n ${ }^{\circ}$ CAAE 51891615.0.0000.5310.

Para a análise dos dados observados, foram utilizados os critérios definidos nos objetivos deste estudo, relacionando as práticas do acolhimento com o que preconiza o MS para a prevenção das DCV, conforme o Plano de Ações Estratégicas para o Enfrentamento das DCNT (BRASIL, 2011), Caderno de Prevenção Clínica de DCV, Cerebrovasculares e Renais (BRASIL, 2006a) e o Caderno de HAS para o SUS (BRASIL, 2006b).

\section{APRESENTAÇÃO E ANÁLISE DOS DADOS}

Observou-se que no acolhimento não eram verificados os sinais vitais dos usuários, com exceção de quando este referia alguma alteração. Dessa forma, foi por meio do que os usuários relatavam de queixas e informações da sua condição, que foi identificado quando em risco de desenvolver DCV.

O local onde foram realizados os acolhimentos é identificado como a sala do acolhimento, é usada para as duas ESF e fica localizado na entrada da unidade. Observou-se que uma sala para acolhimento permite que o profissional consiga realizar uma conversa aberta e uma escuta mais qualificada com o usuário, compreendendo melhor o que este solicita. No entanto, a estrutura física é pequena, e a disposição da mesa não é favorável, pois o profissional senta de costas para o usuário, a frente da mesa é voltada para a parede e não para o usuário. Há uma cadeira para o usuário e se houver acompanhante, este fica em pé. O usuário entra sozinho na sala, ou se for da sua vontade o acompanhante também pode participar do acolhimento. $\mathrm{O}$ acolhimento é realizado por quatro técnicos de enfermagem, sendo que entre eles é realizada uma escala.

O usuário precisa vir até a unidade para fazer o agendamento, com exceção das pessoas acima de 60 anos que podem realizar por telefone ou através da agente comunitária de saúde (ACS), desde que esta faça uma justificativa sobre a impossibilidade do usuário ir até a unidade agendar a sua 
consulta. É possível agendar consulta para outro usuário, desde que resida na mesma casa e saiba qual a queixa da outra pessoa.

Quando o usuário chega à unidade, vai até a recepção para pegar uma ficha numerada e dirigir-se à sala do acolhimento. Todas as pessoas que chegam procurando por atendimento médico na unidade passam pelo acolhimento, com exceção de quem tem consulta marcada para o dia ou precisa de outro tipo de atendimento. $\mathrm{O}$ agendamento de consulta com o enfermeiro e pediatra pode ser realizado na recepção conforme a disponibilidade de horários da agenda de cada profissional. $\mathrm{O}$ atendimento com nutricionista é realizado após encaminhamento pelo médico ou quando o usuário procura esse tipo de atendimento em função de alguma comorbidade. Neste caso, não é necessário ter o encaminhamento do médico e o agendamento pode ser realizado na recepção.

No acolhimento é questionado o motivo pelo qual a pessoa procura a unidade. $\mathrm{O}$ profissional pergunta quais sintomas o usuário apresenta, por quanto tempo, se tem dor, onde é a dor, se já procurou atendimento antes, se tem algum encaminhamento e se está fazendo uso de alguma medicação. Após, é agendada uma consulta com o médico da ESF. Neste momento, o usuário relata suas queixas e aflições, para que o profissional possa programar uma consulta com base no que o usuário relatou. Para avaliar o risco do paciente não é usado protocolo, o agendamento se dá conforme os horários disponíveis e a percepção do usuário sobre o tempo de espera possível para o atendimento.

Uma estratégia que possibilite identificar as diferentes gradações de risco ou situações de maior urgência para priorizar o atendimento e garantir acesso com equidade ao usuário é a adoção da avaliação/ estratificação de risco como um instrumento. Esta pode orientar os profissionais não só com o tipo de intervenção/ oferta de cuidado necessário, como também em relação ao tempo em que isso deve acontecer (BRASIL, 2013b).

Problemas relatados pelo usuário, como: alteração da pressão arterial, diabetes, dislipidemias, infecção no trato urinário, dor, febre, tratamento oncológico, são consideradas prioridades para atendimento médico. Ressaltase que, geralmente, geralmente já tem alguma condição crônica agravada.

O Caderno de Prevenção Clínica de DCV, Cerebrovasculares e Renais (BRASIL, 2006a), refere que mais relevante do que saber o diagnóstico de um usuário, por exemplo, HAS ou diabetes, é verificar o seu risco cardiovascular [...]. Na perspectiva de prevenção, quanto mais elevado o risco de desenvolver DCV, maior deve ser a capacidade de uma intervenção terapêutica ou preventiva.

Foram observados no acolhimento, orientações para verificação da pressão arterial mais vezes na semana até a consulta com o médico, cuidado com alimentos que possam elevar os triglicerídeos, colesterol e diabetes, ingestão de líquidos, e verificado o sinal vital que o usuário referiu estar alterado. No 
entanto, os profissionais não aprofundaram essas orientações, as quais foram superficiais e rápidas. Não se observou registro das orientações realizadas. O único registro que acontece é o lançamento da consulta no sistema. Para que aconteça um melhor acompanhamento e longitudinalidade do cuidado é importante em qualquer UBS, independentemente da modalidade de equipe que nela esteja implantada, fazer o registro dos atendimentos realizados em prontuário individual/familiar (BRASIL, 2013b).

No Plano de Ações Estratégicas para o Enfrentamento das DCNT (BRASIL, 2011), além de garantir o acolhimento e a integralidade da atenção aos portadores de DCNT, é necessário realizar ações de prevenção primária para pessoas em risco de desenvolver DCNT, com vistas à adoção de modos saudáveis de vida, implementando iniciativas como: Academia da Saúde, alimentação saudável, grupos operativos e outros, para suporte no desenvolvimento de hábitos saudáveis de vida.

A demanda de usuários que passam pelo acolhimento é alta. No período em que aconteceu a observação, foram acolhidos, em um turno de trabalho de quatro horas, em média 30 usuários, fazendo com que o tempo seja um limitador para desenvolver esse tipo de aconselhamento. Nas situações em que o usuário não podia esperar por atendimento, a orientação era que o mesmo fosse até a UPA (Unidade de Pronto Atendimento), fizesse a medicação conforme a necessidade e retornasse para programar o acompanhamento com o médico na ESF, a fim de realizar a investigação dos sintomas.

Alguns usuários referiram não conhecer o funcionamento da unidade. Estes entendiam que o atendimento seria por ordem de chegada e de que iriam esperar muito tempo para isto. Esses usuários chegavam no acolhimento e queriam logo ser atendidos. Os técnicos de enfermagem explicavam que primeiro eles passariam pelo acolhimento a fim de verificar a sua necessidade e programar uma consulta. Também era esclarecido que com o acolhimento não existem mais as filas, sendo que o tempo de espera não chegava a 15 minutos e dependendo do horário que o usuário viesse para agendar uma consulta, não encontraria fila de espera. Quando o usuário recebia essa explicação, entendia que o processo de trabalho havia mudado na unidade. Os profissionais explicavam que a ESF trabalha com prevenção e se o usuário precisasse de atendimento imediato deveria se dirigir a UPA.

O processo de trabalho relatado acima mostra que a ESF está centrada na oferta de consulta médica programada. No entanto, entende-se que o acolhimento à demanda espontânea pode e deve abranger as mais diversas demandas dos usuários na UBS, que devem estar disponíveis nesse tipo de serviço. É essencial que as UBS estejam abertas e preparadas para acolher o que não pode ser programado, as eventualidades, os imprevistos. É muito mais acessível para o usuário ir até a unidade do seu bairro do que se deslocar a um pronto- atendimento, além disso, é um momento de criação e fortalecimento de vínculos entre usuário/ profissionais. A UBS, para ser resolutiva, deve ser 
capaz de ampliar a escuta e ter um escopo ampliado de ofertas para lidar com a complexidade de sofrimentos, adoecimentos, demandas e necessidades de saúde às quais as pessoas estão constantemente expostas (BRASIL, 2013b).

A forma pela qual o processo de trabalho da unidade está organizado provoca uma reflexão, pois é centrado na figura do médico. Segundo Franco, Bueno e Merhy (1999, p. 348):

$\mathrm{O}$ acolhimento modifica radicalmente o processo de trabalho. O impacto da reorganização do trabalho na Unidade se dá principalmente sobre os profissionais não-médicos que fazem a assistência. Na atual situação, a equipe de acolhimento passa a ser o centro da atividade no atendimento aos usuários. Os profissionais não-médicos passam a usar todo seu arsenal tecnológico, o conhecimento para a assistência, na escuta e solução de problemas de saúde trazidos pela população usuária dos serviços da Unidade.

Observou-se uma comunicação clara e acessível entre os profissionais e o usuário, assim como um relacionamento baseado no respeito e na tentativa de atender com equidade. Verificou-se que o profissional conhecia o histórico do usuário e de sua família, e dessa forma apresentavam um bom vínculo com o mesmo.

Quando o usuário relatava alguma dúvida no resultado de exames, era encaminhado para a avaliação da enfermeira. No caso de se confirmar a alteração, esta encaminhava ao médico, para definir tratamento ou programar uma consulta.

Os encaminhamentos para alguma especialidade médica ou realização de exames tinha número limitado por mês, filas de espera e dependendo da especialidade médica ou do exame que era preciso realizar, a espera poderia ser longa. Dessa forma, garantir uma atenção resolutiva é uma dificuldade encontrada pelos profissionais. A articulação com outros serviços de saúde, quando necessário, era realizada pelo médico ou pela enfermeira por meio de documento de referência.

Orientações em relação à atividade física, alimentação saudável, tabagismo, álcool e envelhecimento ativo são pouco observadas no acolhimento. Algumas orientações são realizadas em outro momento, quando são verificados os sinais vitais, antes da consulta médica. No entanto, também são orientações rápidas e superficiais, onde o profissional pergunta se o usuário ingere frutas e verduras, orientam para não comer dois carboidratos ao mesmo tempo, cuidar com o uso do sal na comida e praticar exercício físico. Assim como no acolhimento, estas orientações não são registradas.

A unidade vai iniciar o Programa Academia da Saúde, com sede ao lado da unidade e uma profissional de Educação Física contratada com o objetivo de trabalhar com grupos de educação em saúde. Além disso, a unidade tem um 
grupo para reeducação alimentar, onde os usuários são convidados a participar dos encontros. A procura é grande, pois no momento em que um usuário com sobrepeso vem para a consulta é realizado o convite, além de haver cartazes na unidade informando a data de início do grupo. Tanto no acolhimento quanto na sala de sinais vitais, quando o profissional identifica algum fator de risco, o mesmo orienta a participar dos grupos na academia ou do grupo de reeducação alimentar, mas não é regra, se a demanda de atendimentos é grande no dia, essas informações não acontecem.

O Caderno de Prevenção Clínica de DCV, Cerebrovasculares e Renais (BRASIL, 2006a) menciona que a equipe de ESF tem atribuições e competências, e deve atuar, de forma integrada, com níveis de competência bem estabelecidos, na abordagem da avaliação de risco cardiovascular, medidas preventivas primárias.

Conforme os fatores de risco preconizados pelo MS (BRASIL, 2006a) foi possível identificar a HAS como o fator mais presente entre os usuários. O Caderno de HAS para o SUS (BRASIL, 2006b), enfatiza que os profissionais de saúde da rede básica carregam um papel fundamental na implementação de estratégias de controle da hipertensão arterial, não só na definição do diagnóstico clínico e conduta terapêutica, como também envolver o usuário no seguimento e motivação para seu tratamento e educação continuada.

\section{CONSIDERAÇÕES FINAIS}

Os resultados indicaram que o risco de desenvolver DCV é identificado parcialmente no acolhimento, limitado pela demanda do serviço, não constituindo uma rotina. Sugere-se que a equipe, de acordo com a sua realidade, utilize um protocolo que facilite a análise das necessidades dos usuários e a identificação dos usuários em risco de desenvolver DCV.

Observou-se que a abordagem no acolhimento ao usuário com risco de desenvolver DCV fica restrita a programar uma consulta médica. Este fato evidencia a necessidade de não restringir o acolhimento ao agendamento de consultas, mas pensar em outras ofertas e estratégias de cuidado. Nesse sentido, a pesquisa recomenda a reflexão por parte da equipe com relação ao processo de acolhimento e revisão da forma de realizar esta prática.

Os cuidados estabelecidos com o usuário em risco de desenvolver DCV foram de orientação para controle dos fatores de risco e o convite para participação no Programa Academia da Saúde e no grupo de reeducação alimentar. No entanto, observou-se que foram pouco consistentes, realizados de forma superficial, em meio ao atendimento de uma grande demanda.

Um fator limitante deste estudo foi a dificuldade em identificar e incluir os usuários em risco de desenvolver DCV pela forma como era feito o acolhimento. Dos 18 dias de observação deste estudo, foram detectados 59 acolhimentos de pessoas identificadas com risco de desenvolver DCV, sendo que a demanda do 
serviço era grande; no entanto, os sinais vitais não eram verificados e a escuta do profissional era pela queixa do usuário e o agendamento de consulta. Entendese assim, que muitos usuários em risco não foram identificados, e, portanto, não foram estabelecidas estratégias de acompanhamento e tratamento. A busca por resultados diferentes requer a necessidade de transformação do processo de trabalho, buscando o envolvimento de toda a equipe no planejamento das estratégias de cuidado.

Reconhece-se que as limitações desse estudo tornam necessária a realização de outras pesquisas e abordagens sobre o tema. Contudo, espera-se que as questões aqui apresentadas possam contribuir e oportunizar reflexões para qualificar a identificação e acompanhamento dos usuários com risco às condições crônicas na ESF.

\section{REFERÊNCIAS}

BRASIL. Ministério da Saúde. Secretaria de Atenção à Saúde. Departamento de Atenção Básica. Prevenção clínica de doenças cardiovasculares, cerebrovasculares e renais. Brasília: Ministério da Saúde, n. 14 p. 56 2006a. Disponível em: <bvsms.saude. gov.br/bvs/publicacoes/abcad14.pdf>. Acesso em: 05 jun. 2016.

. Ministério da Saúde. Secretaria de Atenção à Saúde. Departamento de Atenção Básica. Hipertensão arterial sistêmica para o Sistema Único de Saúde. Brasília: Ministério da Saúde, p 58, 2006b. Disponível em: <dab.saude.gov.br/docs/ publicacoes/cadernos_ab/abcad15.pdf $>$. Acesso em: 05 jun. 2016.

. Ministério da Saúde. Secretaria de Atenção à Saúde. Política Nacional de Humanização da Atenção e Gestão do SUS. Acolhimento e classificação de risco nos serviços de urgência. Brasília: Ministério da Saúde, p. 56 2009. Disponível em: $<$ http://bvsms.saude.gov.br/bvs/publicacoes/acolhimento_classificaao_risco_ servico_urgencia.pdf>. Acesso em: 05 jun. 2016.

. Ministério da Saúde. Secretaria de Vigilância em Saúde. Departamento de Análise de Situação de Saúde. Plano de ações estratégicas para o enfrentamento das doenças crônicas não transmissíveis (DCNT) no Brasil 2011-2022. Brasília: Ministério da Saúde, p. 160, 2011. Disponível em: <bvsms.saude.gov.br/bvs/publicacoes/plano_ acoes_enfrent_dcnt_2011.pdf $>$. Acesso em: 05 jun. 2016.

. Ministério da Saúde. Secretaria de Atenção à Saúde. Política Nacional de Humanização (PNH). 1 ed. Brasília, 2013a. Disponível em: < http:/ /bvsms.saude.gov. $\mathrm{br} / \mathrm{bvs} /$ publicacoes/politica_nacional_humanizacao_pnh_folheto.pdf $>$. Acesso em: 05 jun. 2016.

. Ministério da Saúde. Secretaria de Atenção à Saúde. Departamento de Atenção Básica. Acolhimento à demanda espontânea. 1. ed. Brasília: Ministério da Saúde, p. 56, 2013b. Disponível em: <http://bvsms.saude.gov.br/bvs/publicacoes/ acolhimento_demanda_espontanea_cab28v1.pdf $>$. Acesso em: 18 mai. 2016. 
. Ministério da Saúde. Secretaria de Atenção à Saúde. Departamento de

Atenção Básica. Estratégias para o cuidado da pessoa com doença crônica. Brasília: Ministério da Saúde, p.162, 2014. Disponível em: <http:/ /bvsms.saude.gov.br/bvs/ publicacoes/estrategias_cuidado_pessoa_doenca_cronica_cab35.pdf $>$. Acesso em: 18 mai. 2016.

GOLDIM, José R. Manual de iniciação à pesquisa em saúde. 2. ed. Porto Alegre: Dacasa, p. 180, 2000.

IBGE. Pesquisa nacional de saúde 2013: percepção do estado de saúde, estilos de vida e doenças crônicas. Rio de Janeiro: IBGE, p. 180, 2014. Disponível em: <http:/ / biblioteca.ibge.gov.br/biblioteca-catalogo?view=detalhes\&id=291110>. Acesso em: 05 jun. 2016.

Estimativa da população, 2015. Disponível em: <http:/ / cidades.ibge.gov.br/ xtras $/$ temas.php?codmun=431140\&idtema=130>. Acesso em: 05 jun. 2016.

LAVILLE, Christian; DIONNE, Jean. A construção do saber: manual de metodologia da pesquisa em ciências humanas. Belo Horizonte: UFMG, 1999.

MALTA, Deborah C. et al. Resultados do monitoramento dos Fatores de risco e Proteção para Doenças Crônicas Não Transmissíveis nas capitais brasileiras por inquérito telefônico, 2008. Rev. bras. epidemiol, v. 15, n. 3, p. 639-650, 2012. Disponível em: <http:/ / www.scielo.br/scielo.php?script=sci_arttext\&pid=S1415790X2012000300017>. Acesso em: 18 mai. 2016.

MATTOS, Luiz A. P. e Racionalidade e métodos: registro da prática clínica em pacientes de alto risco cardiovascular. Arq. bras. cardiol, v. 97, n. 1, p. 3-7, 2011. Disponível em: <http:/ / www.scielo.br/scielo.php?pid=S0066782X2011005000062\&script=sci_arttext>. Acesso em: 18 mai. 2016.

MENDES, Eugênio V. O cuidado das condições crônicas na atenção primária à saúde: o imperativo da consolidação da estratégia da saúde da família. Brasília: Organização Pan-Americana da Saúde, p. 512, 2012. Disponível em: <http:/ /bvsms.saude.gov. $\mathrm{br} / \mathrm{bvs} /$ publicacoes/cuidado_condicoes_atencao_primaria_saude.pdf $>$. Acesso em: 18 mai. 2016.

MERHY, Emerson E.; BUENO, Wanderlei S.; FRANCO, Túlio B.; O acolhimento e os processos de trabalho em saúde: o caso de Betim. Cad. de Saúde Pública, Rio de Janeiro, v. 15, n.2, p. 345-353, 1999. Disponível em: http:/ / www.eeaac.uff.br/ professores/merhy/indexados-16.pdf. Acesso em: 08 jul. 2016. 